\title{
The True Nature of Viruses Elucidated by Systems Virology
}

\author{
Kei Sato* \\ Division of Systems Virology, The Institute of Medical Science, The University of Tokyo, Tokyo, Japan
}

Keywords: systems virology, virology, emerging virus, virome, evolution

\section{WHAT IS "SYSTEMS VIROLOGY"?}

The term "Systems Virology" does not describe a specific aspect of virology, but was coined to cover fundamental and interdisciplinary topics across all aspects of viruses. The word "virus" originates from the Latin term "virus," which means "poison." Today, however, we know that viruses are not necessarily pathogens, but can also be symbionts and important members of ecosystems. With the help of scientific technologies from multiple disciplines, "Systems Virology" can provide key insights into the origins, evolution, mode of action, viral dynamics, and the "raison d'être" of viruses. This includes observations brought from wet-lab experiments at the multiscale level (e.g., single virion, single cell, within host, and population level), phylogenetic analyses, mathematical modeling, bioinformatics and interdisciplinary approaches that combine them. In other words, through a multidisciplinary Systems Virology approach that may help to answer some very basic questions in virology, we aim to get to the very essence of what it means to be a virus:

\section{How Did Viruses Emerge?}

Viruses have driven the evolution of hosts for millions of years, as they are a component of the Earth's ecosystem. The number of viruses on our planet has been estimated to be $10^{31}$. Yet, nobody knows how viruses emerge(d). Sciences addressing the origin of viruses should be important when we think about the origin of lives. "Viral symbiosis" and "viruses in ecosystems" should be keywords in this research area.

In addition to the concerns on the ancient origin of all viruses, the origins of newly emerging viruses should be also considered. The emergence of SARS-CoV-2 has once again illustrated the importance to investigate and understand the origins of viruses. It has been hypothesized that this virus originated from a cross-species transmission of a bat coronavirus to humans. However, it remains unclear how such a spillover event occurred. Thus, surveying the origins of pathogenic viruses is of key importance for human health and society. Moreover, when we think about the Earth's ecosystem and the intersection of human, animal, and environmental health as "One Health," considering viral cross-species transmission should also be important. "Zoonotic infection," "spillover events," and "emerging/re-emerging viruses" should be keywords in this research area. "Molecular phylogenetics" and "molecular evolution" are important to trace viral transmission in the wild and human society, and experiments addressing "virus-host interaction" and "viral pathogenesis" should also be essential.

\section{How Do Viruses Coexist With Their Hosts?}

Received: 04 November 2021
Accepted: 18 November 2021

Published: 14 December 2021

Citation:

Sato K (2021) The True Nature of Viruses Elucidated by Systems Virology. Front. Virol. 1:808865. doi: 10.3389/fviro.2021.808865 categories is not very clear. Moreover, certain viruses integrated their genomes into the host DNA. For instance, $\sim 7 \%$ of the human genome is of retroviral origin. This is direct evidence that hosts (e.g., humans) have coevolved with viruses (e.g., retroviruses). Today, we know that not only retroviruses but also other virus families have integrated their nucleic acids into the host genome. However, it remains largely unclear why these viral genomes were integrated and got 
fixed in the host genome. Understanding "virus-host coevolution" and "endogenous viral elements" should apparently be important to consider the raison d'être of viruses. "Molecular phylogenetics" and "molecular evolution" would be important to trace the evolutionary scenario between viruses and hosts, and the experiments addressing "virus-host interaction" should also be crucial for the in-depth understanding on the interplay between viruses and hosts.

\section{How Many and What Kind of Viruses Are There?}

If there are so many non-pathogenic viruses, what is their role in the human body and their contribution to human health? Before we can fully answer these questions, we need to know what kind of viruses there are in humans. While the importance of the "microbiome" (i.e., commensal bacteria) for human health has been relatively well-described, little is known about the role of commensal viruses. The concept "virome" should be important to understand the raison d'être of viruses. Numerous publicly available next generation sequence data represent a valuable source and starting point to answer these questions, and metagenomic analyses will provide important insights into the identity and role of non-pathogenic viruses in humans and beyond. "Metagenomic analysis" and "bioinformatics" would be useful for addressing this topic.

\section{WHAT IS THE IDENTITY OF VIRUSES?}

The term "virus" is rather generic and somewhat elusive. Let me take "the influenza virus circulating this year" as an example. While its general shape and virion structure is similar to the influenza virus that was circulating "last year," its pathogenicity and infectivity may be entirely different. Moreover, the influenza virus that infected you is not identical to the one that infected me. Finally, the influenza viruses that replicated in my cells yesterday are neither identical to those replicating in me today, nor to those that will be replicating in me tomorrow.

These examples illustrate the difficulty to define and express "the concept of a virus" through the single term "virus." I prefer an image, in which "viruses" are birds flying in swirling masses in the evening sky, or a flock of young fish migrating in the sea. Each individual can be recognized in detail as an entity on its own. At the same time, however, when we try to grasp them as "the whole entity," it is vague and variable.

Then, what makes a virus "a virus"? To answer this question, its shape, infectivity, pathogenicity, and even certain specialized classifications such as genotypes and serotypes would be just sufficient conditions (e.g., apples, watermelons, cherries), however, none of them are necessary conditions (e.g., fruit). So, what are the necessary condition(s) for expressing a virus "the virus," namely, what is "the real" or "the entity" of the virus?

"Systems Virology" is a discipline that aims to answer basic questions on viruses such as those mentioned above through advanced scientific interrogation. "Systems Virology" will shed light on a variety of aspects of viruses that have not yet been fully addressed and represents a new field. I welcome you all to contribute to the future of virology.

\section{AUTHOR CONTRIBUTIONS}

KS wrote the manuscript.

\section{ACKNOWLEDGMENTS}

The author thanks Drs. Daniel Sauter (University Hospital Tübingen, Germany) and Alex A. Compton (National Cancer Institute, USA) for kind feedback on this manuscript.

Conflict of Interest: The author declares that the research was conducted in the absence of any commercial or financial relationships that could be construed as a potential conflict of interest.

Publisher's Note: All claims expressed in this article are solely those of the authors and do not necessarily represent those of their affiliated organizations, or those of the publisher, the editors and the reviewers. Any product that may be evaluated in this article, or claim that may be made by its manufacturer, is not guaranteed or endorsed by the publisher.

Copyright (c) 2021 Sato. This is an open-access article distributed under the terms of the Creative Commons Attribution License (CC BY). The use, distribution or reproduction in other forums is permitted, provided the original author(s) and the copyright owner(s) are credited and that the original publication in this journal is cited, in accordance with accepted academic practice. No use, distribution or reproduction is permitted which does not comply with these terms. 